\title{
Novel copolymers of vinyl acetate. 3 Ring-substituted ethyl 2-cyano-3- phenyl-2-propenoates
}

Kamil M. Wojdyla, Benjamin Y. Killam, Carrie L. Grady, Faheem Jesani, Anne M.

Johnson, Vickie Kalamaras, Jesse J. Kong, Kiersten L. Thompson, Charles A. Rallo, Anna L. Lampignano, Meenakshi Malhotra, Sarah Mikach and Gregory B. Kharas

DePaul University, Chemistry and Biochemistry Department, 1110 West Belden Avenue, Chicago, IL 60614-3214

\begin{abstract}
Novel copolymers of vinyl acetate and ring-substituted ethyl 2-cyano-3-phenyl-2-

propenoates, $\mathrm{RPhCH}=\mathrm{C}(\mathrm{CN}) \mathrm{CO}_{2} \mathrm{C}_{2} \mathrm{H}_{5}$ (where $\mathrm{R}$ is 4-acetoxy, 4-acetamido, 2-cyano, 3cyano, 4-cyano, 4-dimethylamino, 4-diethylamino, 2,4,6-trimethyl, 2,3-dimethyl-4methoxy, 2,4-dimethoxy-3-methyl were prepared in solution with radical initiation at $70^{\circ} \mathrm{C}$. The propenoates were synthesized by the piperidine catalyzed Knoevenagel condensation of ring-substituted benzaldehydes and ethyl cyanoacetate, and characterized by $\mathrm{CHN}$ analysis, IR, ${ }^{1} \mathrm{H}$ and ${ }^{13} \mathrm{C}-\mathrm{NMR}$. The compositions of the copolymers were calculated from nitrogen analysis and the structures were analyzed by IR, ${ }^{1} \mathrm{H}$ and ${ }^{13} \mathrm{C}-\mathrm{NMR}$. Thermal behavior of the copolymers was studied by DSC (Tg) and TGA. Decomposition of the copolymers in
\end{abstract}


nitrogen occurred in two steps, first in the $160-350^{\circ} \mathrm{C}$ range with residue $(1.5-15.1 \mathrm{wt} \%)$, which then decomposed in the $500-650^{\circ} \mathrm{C}$ range.

\section{Introduction}

Electrophilic aryl-functionalized trisubstituted ethylenes, ethyl 2-cyano-3-phenyl-2propenoates, $\mathrm{RPhCH}=\mathrm{C}(\mathrm{CN}) \mathrm{CO}_{2} \mathrm{C}_{2} \mathrm{H}_{5}(\mathrm{ECPP})$ continue to attract attention as organic intermediates and compounds with useful properties. Thus, acetoxy ring-substituted ECPP was used as intermediate in microwave-promoted sequential three-component synthesis of tetrahydrobenzo[b]pyran in water catalyzed by heterogeneous amine grafted on silica [1], in Knoevenagel reactions catalyzed by nano-silica PAMAM dendrimer [2] and by amine supported on silica gel [3].

4-Acetamido ECPP was involved in studies of enaminones in heterocyclic syntheses as a new one-step synthetic route to pyrrolo[3,4-b]pyridine and convenient syntheses of 1,4dihydropyridines and 1,1'-(1,4-phenylene)bis(1,4-dihydropyridine) [4]. It was also used as nonlinear optical material based on cyano(acetylamino)cinnamate ester [5]. 2-Cyano ECPP was used in studies of structure-activity relationships and brain uptake of a novel series of benzopyran inhibitors of insulin-regulated aminopeptidase [6]. 3-Cyano ECPP was involved in AgNO3-catalysed intramolecular cyclization leading to functionalized cyclopentanones and spiro-cyclopentanones [7]. It also was reported in studies of adenine-based coordination polymers [8]. 4-Cyano ECPP is reported in synthesis of 1,7bis (N-substituted-aminomethyl)-2,8-dihydroxy-troger's bases and their application in Aldol-Ullmann reaction [9] and also in studies on synthesis, antitumor activity and molecular docking of seven novel thiazacridine derivatives [10]. 4-Dimethylamino ECPP 
was reported in synthesis of 1-[(aryl)(3-amino-5-oxopyrazolidin-4-ylidene) methyl]-2oxo-1,2-dihydroquinoline-3-carboxylic acid derivatives and studies of their breast anticancer activity [11]. 4-Diethylamino ECPP was mentioned in the report on design, synthesis and vasorelaxant evaluation of novel coumarin-pyrimidine hybrids [12]. 2,4,6trimethyl ECPP is reported in catalyst study of the Knoevenagel condensation [13]. In continuation of our research on radical copolymerization of vinyl acetate and arylsubstituted esters 2-cyano-3-phenyl-2-propenoic acid, we have prepared ring-substituted ethyl 2-cyano-3-phenyl-2-propenoates, $\mathrm{RPhCH}=\mathrm{C}(\mathrm{CN}) \mathrm{CO}_{2} \mathrm{C}_{2} \mathrm{H}_{5}$ (where $\mathrm{R}$ is 4-acetoxy, 4acetamido, 2-cyano, 3-cyano, 4-cyano, 4-dimethylamino, 4-diethylamino, 2,4,6-trimethyl, 2,3-dimethyl-4-methoxy, 2,4-dimethoxy-3-methyl) and copolymerized them with vinyl acetate. There are reports of syntheses of 4-acetoxy, 4-acetamido, 2-cyano, 3-cyano, 4cyano, 4-dimethylamino, 4-diethylamino ECPP and copolymerization with styrene [14]. There are no reports on synthesis of 2,3-dimethyl-4-methoxy and 2,4-dimethoxy-3methyl ECPP. To the best of our knowledge, there have been no reports on copolymerization of these ethyl 2-cyano-3-phenyl-2-propenoates with vinyl acetate [15].

\section{Experimental}

4-Acetoxy, 4-acetamido, 2-cyano, 3-cyano, 4-cyano, 4-dimethylamino, 4-diethylamino, 2,4,6-trimethyl, 2,3-dimethyl-4-methoxy, 2,4-dimethoxy-3-methyl-substituted benzaldehydes, ethyl cyanoacetate, piperidine, vinyl acetate, 1,1'azobiscyclohexanecarbonitrile, $(\mathrm{ABCN})$, and toluene supplied from Sigma-Aldrich Co., were used as received. Instrumentation is described in [16]. 


\section{Synthesis of Monomers}

The ring-substituted ethyl 2-cyano-3-phenyl-2-propenoates (ECPP) were synthesized by Knoevenagel condensation [17] of a ring-substituted benzaldehyde with ethyl cyanoacetate, catalyzed by base, piperidine. The preparation procedure was essentially the same for all the monomers [16].

$$
\mathrm{RPhCHO}+\mathrm{NCCH}_{2} \mathrm{CO}_{2} \mathrm{C}_{2} \mathrm{H}_{5} \rightarrow \mathrm{RPhCH}=\mathrm{C}(\mathrm{CN}) \mathrm{CO}_{2} \mathrm{C}_{2} \mathrm{H}_{5}
$$

where $\mathrm{R}$ is 4-acetoxy, 4-acetamido, 2-cyano, 3-cyano, 4-cyano, 4-dimethylamino, 4diethylamino, 2,4,6-trimethyl, 2,3-dimethyl-4-methoxy, 2,4-dimethoxy-3-methyl.

\subsection{Ethyl 2-cyano-3-(4-acetoxyphenyl)-2-propenoate}

Yield $87 \%$; mp $95.3^{\circ} \mathrm{C},{ }^{1} \mathrm{H}-\mathrm{NMR} \delta 8.2$ (s, $\left.1 \mathrm{H}, \mathrm{CH}=\right), 7.5,6.9$ (m, 4H, Ph), 4.3 (q, 2H,

$\left.\mathrm{OCH}_{2}\right), 2.2\left(\mathrm{~s}, 3 \mathrm{H}, \mathrm{PhCO}_{2} \mathrm{CH}_{3}\right), 1.3\left(\mathrm{t}, 3 \mathrm{H}, \mathrm{OCH}_{2} \underline{\mathrm{C}}_{3}\right) ;{ }^{13} \mathrm{C}-\mathrm{NMR} \delta 163,162(\mathrm{C}=\mathrm{O}), 152$ $(\mathrm{HC}=), 148,131,124,121(\mathrm{Ph}), 116(\mathrm{CN}), 111(\mathrm{C}=), 61\left(\mathrm{OCH}_{2}\right), 21\left(\mathrm{PhO}_{2} \mathrm{CH}_{3}\right), 14$ $\left(\mathrm{OCH}_{2} \underline{\mathrm{CH}}_{3}\right)$; IR $\left(\mathrm{cm}^{-1}\right)$ : 3023-2832 (m, C-H), 2224 (m, CN), 1742 (s, C=O), 1573 (C=C), 1232 (s, C-O-CH3), 811 (s, C-H out of plane). Anal. Calcd. for $\mathrm{C}_{14} \mathrm{H}_{13} \mathrm{NO}_{4}: \mathrm{C}, 64.86 ; \mathrm{H}$, 5.05; N, 5.40; Found: C, 64.82; H, 5.10; N, 5.36.

\subsection{Ethyl 2-cyano-3-(4-acetamidophenyl)-2-propenoate}

Yield $84 \%$; mp $195.8^{\circ} \mathrm{C},{ }^{1} \mathrm{H}-\mathrm{NMR} \delta 8.2(\mathrm{~s}, 1 \mathrm{H}, \mathrm{CH}=), 8.0,7.6(\mathrm{~m}, 4 \mathrm{H}, \mathrm{Ph}), 5.2(\mathrm{~s}, 1 \mathrm{H}$, $\mathrm{NH}), 4.3\left(\mathrm{q}, 2 \mathrm{H}, \mathrm{OCH}_{2}\right), 2.1\left(\mathrm{~s}, 3 \mathrm{H}, \mathrm{PhNHCOCH}_{3}\right), 1.3\left(\mathrm{t}, 3 \mathrm{H}, \mathrm{OCH}_{2} \mathrm{CH}_{3}\right) ;{ }^{13} \mathrm{C}-\mathrm{NMR} \delta$ 168, $163(\mathrm{C}=\mathrm{O}), 154(\mathrm{HC}=), 151,134,120,119(\mathrm{Ph}), 116(\mathrm{CN}), 96(\mathrm{C}=), 61\left(\mathrm{OCH}_{2}\right), 23$ $\left(\mathrm{PhCOCH}_{3}\right), 14\left(\mathrm{OCH}_{2} \underline{C H}_{3}\right)$; IR $\left(\mathrm{cm}^{-1}\right):$ 3038-2833 (m, C-H), 2223 (m, CN), 1722 (s, 
$\mathrm{C}=\mathrm{O}), 1582(\mathrm{C}=\mathrm{C}), 1221\left(\mathrm{~s}, \mathrm{C}-\mathrm{O}-\mathrm{CH}_{3}\right), 841$ (s, C-H out of plane). Anal. Calcd. for $\mathrm{C}_{14} \mathrm{H}_{14} \mathrm{~N}_{2} \mathrm{O}_{3}: \mathrm{C}, 65.11 ; \mathrm{H}, 5.46 ; \mathrm{N}, 10.85$; Found: C, 65.67; H, 5.46; N, 10.84.

\subsection{Ethyl 2-cyano-3-(2-cyanophenyl)-2-propenoate}

Yield $89 \%$; mp $223.4^{\circ} \mathrm{C},{ }^{1} \mathrm{H}-\mathrm{NMR} \delta 8.2$ (s, $\left.1 \mathrm{H}, \mathrm{CH}=\right), 7.9-7.0(\mathrm{~m}, 4 \mathrm{H}, \mathrm{Ph}), 4.3(\mathrm{q}, 2 \mathrm{H}$,

$\left.\mathrm{OCH}_{2}\right), 1.3\left(\mathrm{t}, 3 \mathrm{H}, \mathrm{OCH}_{2} \mathrm{CH}_{3}\right) ;{ }^{13} \mathrm{C}-\mathrm{NMR} \delta 163(\mathrm{C}=\mathrm{O}), 152(\mathrm{HC}=), 147,136,133,131$,

$130(\mathrm{Ph}), 117,116(\mathrm{CN}), 100(\mathrm{C}=), 61\left(\mathrm{OCH}_{2}\right), 14\left(\mathrm{OCH}_{2} \underline{\mathrm{CH}}_{3}\right) ; \mathrm{IR}\left(\mathrm{cm}^{-1}\right): 3021-2922$

(m, C-H), 2223 (m, CN), 1742 (s, C=O), 1232 (s, C-O-C), 843 (s, C-H out of plane).

Anal. Calcd. for $\mathrm{C}_{13} \mathrm{H}_{10} \mathrm{~N}_{2} \mathrm{O}_{2}$ : C, 69.02; H, 4.46; N, 12.38; Found: C, 63.85; H, 4.45; N, 12.40 .

\subsection{Ethyl 2-cyano-3-(3-cyanophenyl)-2-propenoate}

Yield $76 \%$; mp $128.8^{\circ} \mathrm{C},{ }^{1} \mathrm{H}-\mathrm{NMR} \delta 8.2(\mathrm{~s}, 1 \mathrm{H}, \mathrm{CH}=), 7.7-7.0(\mathrm{~m}, 4 \mathrm{H}, \mathrm{Ph}), 4.3(\mathrm{q}, 2 \mathrm{H}$, $\left.\mathrm{OCH}_{2}\right), 1.3\left(\mathrm{t}, 3 \mathrm{H}, \mathrm{OCH}_{2} \underline{\mathrm{CH}}_{3}\right) ;{ }^{13} \mathrm{C}-\mathrm{NMR} \delta 163(\mathrm{C}=\mathrm{O}), 154(\mathrm{HC}=), 136,129,113(\mathrm{Ph})$, 119, $116(\mathrm{CN}), 103(\mathrm{C}=), 61\left(\mathrm{OCH}_{2}\right), 14\left(\mathrm{OCH}_{2} \underline{\mathrm{CH}_{3}}\right)$; IR $\left(\mathrm{cm}^{-1}\right): 3124-2825(\mathrm{~m}, \mathrm{C}-\mathrm{H})$, 2222 (m, CN), 1724 (s, C=O), 1618 (C=C), 1242 (s, C-O-C), 849 (s, C-H out of plane). Anal. Calcd. for $\mathrm{C}_{13} \mathrm{H}_{10} \mathrm{~N}_{2} \mathrm{O}_{2}$ : C, 69.02; H, 4.46; N, 12.38; Found: C, 69.05; H, 4.27; N, 12.37.

\subsection{Ethyl 2-cyano-3-(4-cyanophenyl)-2-propenoate}

Yield 82\%; ${ }^{1} \mathrm{H}-\mathrm{NMR} \delta 8.2(\mathrm{~s}, 1 \mathrm{H}, \mathrm{CH}=), 7.9,7.8(\mathrm{~m}, 4 \mathrm{H}, \mathrm{Ph}), 4.3\left(\mathrm{q}, 2 \mathrm{H}, \mathrm{OCH}_{2}\right), 1.3(\mathrm{t}$, $\left.3 \mathrm{H}, \mathrm{OCH}_{2} \mathrm{CH}_{3}\right) ;{ }^{13} \mathrm{C}-\mathrm{NMR} \delta 163(\mathrm{C}=\mathrm{O}), 154(\mathrm{HC}=), 136,133,131,118(\mathrm{Ph}), 116(\mathrm{CN})$, $113(\mathrm{C}=), 61\left(\mathrm{OCH}_{2}\right), 14\left(\mathrm{OCH}_{2} \underline{\mathrm{CH}}_{3}\right) ; \mathrm{IR}\left(\mathrm{cm}^{-1}\right): 3031-2857$ (m, C-H), $2223(\mathrm{~m}, \mathrm{CN})$, 
1736 (s, C=O), $1611(\mathrm{C}=\mathrm{C}), 1243$ (s, C-O-C), 842 (s, C-H out of plane). Anal. Calcd. for $\mathrm{C}_{13} \mathrm{H}_{10} \mathrm{~N}_{2} \mathrm{O}_{2}$ : C, 69.02; H, 4.46; N, 12.38; Found: C, 69.01; H, 4.17; N, 12.27.

\subsection{Ethyl 2-cyano-3-(4-dimethylaminophenyl)-2-propenoate}

Yield $72 \%$; mp $130.2{ }^{\circ} \mathrm{C}$; ${ }^{1} \mathrm{H}-\mathrm{NMR} \delta 8.2$ (s, $\left.1 \mathrm{H}, \mathrm{CH}=\right), 7.6,7.0$ (m, 4H, Ph), 4.3 (q, 2H, $\left.\mathrm{OCH}_{2}\right), 3.0\left(\mathrm{~s}, 6 \mathrm{H}, \mathrm{N}\left(\mathrm{CH}_{3}\right)_{2}, 1.3\left(\mathrm{~m}, 3 \mathrm{H}, \mathrm{CH}_{3}\right) ;{ }^{13} \mathrm{C}-\mathrm{NMR} \delta 163(\mathrm{C}=\mathrm{O}), 154(\mathrm{HC}=), 152\right.$, 134, 121, $111(\mathrm{Ph}), 61\left(\mathrm{OCH}_{2}\right), 40\left(\mathrm{PhNCH}_{3}\right), 14\left(\mathrm{OCH}_{2} \underline{\mathrm{CH}}_{3}\right) ; \mathrm{IR}\left(\mathrm{cm}^{-1}\right): 3005-2885(\mathrm{~m}$, C-H), 2223 (m, CN), 1723 (s, C=O), 1581 (C=C), 1221 (s, C-O-C), 843 (s, C-H out of plane). Anal. Calcd. for $\mathrm{C}_{14} \mathrm{H}_{16} \mathrm{~N}_{2} \mathrm{O}_{2}$ : C, 68.83; H, 6.60; N, 11.47; Found: C, 69.02; H, $6.60 ; \mathrm{N}, 11.40$.

\subsection{Ethyl 2-cyano-3-(4-diethylaminophenyl)-2-propenoate}

Yield $85 \%$; mp $99.6^{\circ} \mathrm{C} ;{ }^{1} \mathrm{H}-\mathrm{NMR} \delta 8.2(\mathrm{~s}, 1 \mathrm{H}, \mathrm{CH}=), 7.6,7.0(\mathrm{~m}, 4 \mathrm{H}, \mathrm{Ph}), 4.3(\mathrm{q}, 2 \mathrm{H}$, $\left.\mathrm{OCH}_{2}\right), 3.4\left(\mathrm{~s}, 4 \mathrm{H}, \mathrm{NCH}_{2}, 1.3\left(\mathrm{t}, 3 \mathrm{H}, \mathrm{OCH}_{2} \underline{\mathrm{C}}_{3}\right), 1.2\left(\mathrm{t}, 6 \mathrm{H}, \mathrm{NCH}_{2} \mathrm{CH}_{3}\right) ;{ }^{13} \mathrm{C}-\mathrm{NMR} \delta 163\right.$ $(\mathrm{C}=\mathrm{O}), 154(\mathrm{HC}=), 151,134,120,111(\mathrm{Ph}), 116(\mathrm{CN}), 96(\mathrm{C}=), 61\left(\mathrm{OCH}_{2}\right), 44\left(\mathrm{NCH}_{2}\right)$, $14\left(\mathrm{OCH}_{2} \underline{\mathrm{CH}_{3}}\right), 13\left(\mathrm{NCH}_{2} \underline{\mathrm{CH}_{3}}\right) ; \mathrm{IR}\left(\mathrm{cm}^{-1}\right): 3024-2821$ (m, C-H), $2222(\mathrm{~m}, \mathrm{CN}), 1742$ (s, $\mathrm{C}=\mathrm{O}), 1576(\mathrm{C}=\mathrm{C}), 1221$ (s, C-O-C), 845 (s, C-H out of plane). Anal. Calcd. for $\mathrm{C}_{16} \mathrm{H}_{20} \mathrm{~N}_{2} \mathrm{O}_{2}$ : C, 70.56; H, 7.40; N, 10.29; Found: C, 70.73; H, 7.32; N, 10.22.

\subsection{Ethyl 2-cyano-3-(2,4,6-trimethylphenyl)-2-propenoate}

Yield 79\%; ${ }^{1} \mathrm{H}-\mathrm{NMR} \delta 8.1(\mathrm{~s}, 1 \mathrm{H}, \mathrm{CH}=), 6.9(\mathrm{~s}, 2 \mathrm{H}, \mathrm{Ph}), 4.3\left(\mathrm{q}, 2 \mathrm{H}, \mathrm{OCH}_{2}\right), 2.3(\mathrm{~m}, 9 \mathrm{H}$, $\left.\mathrm{PhCH}_{3}\right), 1.3\left(\mathrm{t}, 3 \mathrm{H}, \mathrm{OCH}_{2} \mathrm{CH}_{3}\right) ;{ }^{13} \mathrm{C}-\mathrm{NMR} \delta 163(\mathrm{C}=\mathrm{O}), 152(\mathrm{HC}=), 131,129,125(\mathrm{Ph})$, $116(\mathrm{CN}), 104(\mathrm{C}=), 61\left(\mathrm{OCH}_{2}\right), 20,21\left(\mathrm{PhCH}_{3}\right), 14\left(\mathrm{OCH}_{2} \underline{\mathrm{CH}_{3}}\right) ; \mathrm{IR}\left(\mathrm{cm}^{-1}\right): 3021-2845$ (m, C-H), $2223(\mathrm{~m}, \mathrm{CN}), 1724$ (s, C=O), $1583(\mathrm{C}=\mathrm{C}), 1233$ (s, C-O-CH 3$), 840$ (s, C-H 
out of plane). Anal. Calcd. for $\mathrm{C}_{15} \mathrm{H}_{17} \mathrm{NO}_{2}$ : C, 74.05; H, 7.04; N, 5.76; Found: C, 73.82;

H, 7.06; N, 5.71 .

\subsection{Ethyl 2-cyano-3-(2,3-dimethyl-4-methoxyphenyl)-2-propenoate}

Yield $84 \%$; mp $129.8^{\circ} \mathrm{C},{ }^{1} \mathrm{H}-\mathrm{NMR} \delta 8.1$ (s, $\left.1 \mathrm{H}, \mathrm{CH}=\right), 7.5,7.1(\mathrm{~m}, 2 \mathrm{H}, \mathrm{Ph}), 4.3(\mathrm{q}, 2 \mathrm{H}$,

$\left.\mathrm{OCH}_{2}\right), 3.8\left(\mathrm{~s}, 3 \mathrm{H}, \mathrm{PhOCH}_{3}\right), 2.2,2.3\left(\mathrm{~m}, 6 \mathrm{H}, \mathrm{PhCH}_{3}\right), 1.3\left(\mathrm{t}, 3 \mathrm{H}, \mathrm{OCH}_{2} \mathrm{CH}_{3}\right) ;{ }^{13} \mathrm{C}-\mathrm{NMR}$

$\delta 163(\mathrm{C}=\mathrm{O}), 152(\mathrm{HC}=), 151,131,126,114(\mathrm{Ph}), 116(\mathrm{CN}), 100(\mathrm{C}=), 61\left(\mathrm{OCH}_{2}\right), 56$

$\left(\mathrm{PhOCH}_{3}\right), 14\left(\mathrm{OCH}_{2} \underline{\mathrm{CH}}_{3}\right), 17,11\left(\mathrm{PhCH}_{3}\right)$; IR $\left(\mathrm{cm}^{-1}\right)$ : 3022-2842 (m, C-H), 2224 (m,

$\mathrm{CN}), 1731$ (s, C=O), $1565(\mathrm{C}=\mathrm{C}), 1235$ (s, C-O-CH 3$), 841$ (s, C-H out of plane). Anal.

Calcd. for $\mathrm{C}_{15} \mathrm{H}_{17} \mathrm{NO}_{3}$ : C, 69.48; H, 6.61; N, 5.40; Found: C, 69.44; H, 6.53; N, 5.20.

\subsection{Ethyl 2-cyano-3-(2,4-dimethoxy-3-methylphenyl)-2-propenoate}

Yield $86 \%$; mp $110.7^{\circ} \mathrm{C},{ }^{1} \mathrm{H}-\mathrm{NMR} \delta 8.2(\mathrm{~s}, 1 \mathrm{H}, \mathrm{CH}=), 7.5,7.1(\mathrm{~m}, 2 \mathrm{H}, \mathrm{Ph}), 4.3(\mathrm{q}, 2 \mathrm{H}$, $\left.\mathrm{OCH}_{2}\right), 3.8\left(\mathrm{~s}, 6 \mathrm{H}, \mathrm{PhOCH}_{3}\right), 2.3\left(\mathrm{~m}, 3 \mathrm{H}, \mathrm{PhCH}_{3}\right), 1.3\left(\mathrm{t}, 3 \mathrm{H}, \mathrm{OCH}_{2} \mathrm{CH}_{3}\right) ;{ }^{13} \mathrm{C}-\mathrm{NMR} \delta$ $163(\mathrm{C}=\mathrm{O}), 152(\mathrm{HC}=), 143,131,114,113,108(\mathrm{Ph}), 116(\mathrm{CN}), 97(\mathrm{C}=), 61\left(\mathrm{OCH}_{2}\right), 56$ $\left(\mathrm{PhOCH}_{3}\right), 14\left(\mathrm{OCH}_{2} \underline{\mathrm{CH}}_{3}\right), 8\left(\mathrm{PhCH}_{3}\right) ; \mathrm{IR}\left(\mathrm{cm}^{-1}\right): 3001-2832(\mathrm{~m}, \mathrm{C}-\mathrm{H}), 2222(\mathrm{~m}, \mathrm{CN})$, $1727(\mathrm{~s}, \mathrm{C}=\mathrm{O}), 1576(\mathrm{C}=\mathrm{C}), 1232$ (s, C-O-CH$), 847$ (s, C-H out of plane). Anal. Calcd. for $\mathrm{C}_{15} \mathrm{H}_{17} \mathrm{NO}_{4}$ : C, 65.44; H, 6.22; N, 5.09; Found: C, 65.76; H, 6.27; N, 5.09.

\section{Copolymerization}

An attempted radical homopolymerization of the ECPP compounds did not produced polymeric products similarly to other ring-substituted ECPP [18]. Homopolymerization of vinyl acetate yielded 28.7 of poly(vinyl acetate) under these conditions [18]. 
Copolymerization (Sch. 1) of VAC and the ring-substituted ECPP resulted in formation of copolymers (Table 1) with weight-average molecular masses 8.8 to $12.6 \mathrm{kD}$.

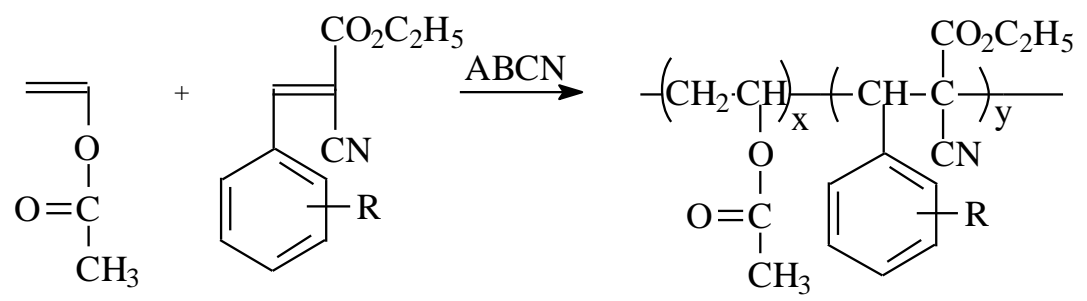

Scheme 1. Copolymerization of vinyl acetate and the ring-substituted ethyl 2-cyano-3phenyl-2-propenoates, $\mathrm{RPhCH}=\mathrm{C}(\mathrm{CN}) \mathrm{CO}_{2} \mathrm{C}_{2} \mathrm{H}_{5}$, where $\mathrm{R}$ is 4-acetoxy, 4-acetamido, 2cyano, 3-cyano, 4-cyano, 4-dimethylamino, 4-diethylamino, 2,4,6-trimethyl, 2,3-dimethyl4-methoxy, 2,4-dimethoxy-3-methyl.

According to the nitrogen elemental analysis, between 42.1 and $49.5 \mathrm{~mol} \%$ of TSE monomer is present in the copolymers prepared at VAC/ECPP $=3(\mathrm{~mol})$, which is indicative of relatively high reactivity of the monomers towards VAC. 
Table 1. Copolymerization of vinyl acetate and ring-substituted ethyl 2-cyano-3-phenyl2-propenoates.

\begin{tabular}{|c|c|c|c|c|c|c|c|c|c|}
\hline & & & & & & \multicolumn{4}{|c|}{$T G A$} \\
\hline $\mathrm{R}$ & $\begin{array}{l}\text { Yield }^{\mathrm{a}} \\
\text { wt } \%\end{array}$ & $\begin{array}{c}\mathrm{N} \\
\mathrm{wt} \%\end{array}$ & $\begin{array}{l}\text { ECCP } \\
\text { in } \\
\text { pol., } \\
\text { mol\% }\end{array}$ & $\begin{array}{l}\mathrm{M}_{\mathrm{w}} \\
\mathrm{kD}\end{array}$ & $\begin{array}{l}\mathrm{T}_{\mathrm{g}} \\
{ }^{\circ} \mathrm{C}\end{array}$ & $\begin{array}{l}\text { Onset of } \\
\text { decomp. } \\
{ }^{\circ} \mathrm{C}\end{array}$ & $\begin{array}{c}10 \% \\
\text { wt } \\
\text { loss, } \\
{ }^{\circ} \mathrm{C}\end{array}$ & $\begin{array}{l}50 \% \\
\text { wt } \\
\text { loss, } \\
{ }^{\circ} \mathrm{C}\end{array}$ & $\begin{array}{c}\text { Residue } \\
\text { at } \\
500^{\circ} \mathrm{C}, \\
\text { wt } \%\end{array}$ \\
\hline 4-Acetoxy & 62.1 & 4.01 & 48.8 & 12.6 & 102 & 216 & 277 & 340 & 12.3 \\
\hline p-Acetamido & 45.9 & 8.10 & 49.5 & 8.8 & 96 & 206 & 255 & 334 & 12.6 \\
\hline 2-Cyano & 57.2 & 8.13 & 42.1 & 9.2 & 98 & 159 & 249 & 341 & 14.1 \\
\hline 3-Сyano & 51.2 & 8.26 & 43.2 & 10.4 & 96 & 150 & 201 & 245 & 9.6 \\
\hline 4-Cyano & 49.3 & 8.23 & 43.0 & 11.1 & 89 & 153 & 208 & 254 & 9.5 \\
\hline $\begin{array}{l}\text { 4- } \\
\text { Dimethylamino }\end{array}$ & 54.2 & 8.17 & 46.6 & 9.1 & 88 & 190 & 256 & 301 & 9.2 .4 \\
\hline $\begin{array}{l}\text { 4- } \\
\text { Diethylamino }\end{array}$ & 68.4 & 7.61 & 47.3 & 10.4 & 94 & 193 & 257 & 301 & 1.5 \\
\hline 2,4,6-trimethyl & 76.3 & 3.92 & 43.0 & 9.8 & 101 & 147 & 201 & 323 & 11.9 \\
\hline $\begin{array}{l}\text { 2,3-Dimethyl- } \\
\text { 4-methoxy }\end{array}$ & 45.4 & 3.91 & 46.5 & 11.4 & 98 & 186 & 246 & 279 & 7.2 \\
\hline $\begin{array}{l}\text { 2,4- } \\
\text { Dimethoxy-3- } \\
\text { methyl }\end{array}$ & 54.2 & 3.67 & 44.7 & 12.5 & 102 & 177 & 223 & 327 & 15.1 \\
\hline
\end{tabular}

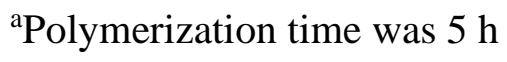




\section{Structure and Thermal Properties}

The structure of VAC-ECPP copolymers was characterized by IR and NMR spectroscopy. A comparison of the spectra of the monomers, copolymers, and polyvinyl acetate with the spectra of ring-unsubstituted ethyl 2-cyano-3-phenyl-2-propenoate - VAC [19] shows, that the reaction between the ECPP monomers and VAC is a copolymerization.

The copolymers prepared in the present work are all soluble in ethyl acetate, THF, DMF and $\mathrm{CHCl}_{3}$ and insoluble in methanol, ethyl ether, and petroleum ether. They are amorphous and show no crystalline DSC endotherm. Results of thermal analysis of VACECPP copolymers are presented in Table 1 . Relatively high $T_{g}$ of the copolymers (88-102 ${ }^{\circ} \mathrm{C}$ ) in comparison with that of polyvinyl acetate, $T_{g}=28-31^{\circ} \mathrm{C}[20]$ indicates decrease of chain mobility of the copolymer due to the high dipolar character of the ECPP structural units.

Information on the degradation of the copolymers was obtained from thermogravimetric analysis. Decomposition of the copolymers in nitrogen occurred in two steps, apparently due to acetic acid elimination [21] in $150-340^{\circ} \mathrm{C}$ range followed by more slow decomposition of formed residue (15.1-1.5 wt $\%$ ), which then decomposed in the $500-650^{\circ} \mathrm{C}$ range.

\section{Conclusions}

Ring-substituted ethyl 2-cyano-3-phenyl-2-propenoates, $\mathrm{RPhCH}=\mathrm{C}(\mathrm{CN}) \mathrm{CO}_{2} \mathrm{C}_{2} \mathrm{H}_{5}$ ( $\mathrm{R}$ is 4acetoxy, 4-acetamido, 2-cyano, 3-cyano, 4-cyano, 4-dimethylamino, 4-diethylamino, 2,4,6trimethyl, 2,3-dimethyl-4-methoxy, 2,4-dimethoxy-3-methyl) were prepared and copolymerized with vinyl acetate. The compositions of the copolymers were calculated from nitrogen analysis and the structures were analyzed by IR, $\mathrm{H}^{1}$ and ${ }^{13} \mathrm{C}-\mathrm{NMR}$. Thermal 
gravimetric analysis indicated that the copolymers decompose in in two steps, first in the $200-500^{\circ} \mathrm{C}$ range with residue (13-16 wt \%), which then decomposed in the $500-650^{\circ} \mathrm{C}$ range.

\section{Acknowledgments}

The authors are grateful to acknowledge that the project was partly supported by the Coatings Industry Education Fund and Chicago Society of Coating Technology.

\section{References}

[1] Microwave-promoted sequential three-component synthesis of tetrahydrobenzo[b]pyran in water catalyzed by heterogeneous amine grafted on silica. Hagiwara, Hisahiro; Numamae, Ayuko; Isobe, Kohei; Hoshi, Takashi; Suzuki, Toshio. Heterocycles (2006), 68(5), 889-895.

[2] Nano-silica PAMAM dendrimer as a novel catalyst for Knoevenagel reactions. Hagiwara, Hisahiro; Sekifuji, Masayoshi; Tsubokawa, Norio; Hoshi, Takashi; Suzuki, Toshio. Chemistry Letters (2009), 38(9), 926-927.

[3] Knoevenagel reaction in water catalyzed by amine supported on silica gel. Isobe, Kohei; Hoshi, Takashi; Suzuki, Toshio; Hagiwara, Hisahiro. Molecular Diversity (2005), 9(4), 317-320

[4] Enaminones in heterocyclic syntheses: Part 4. A new one-step synthetic route to pyrrolo[3,4-b]pyridine and convenient syntheses of 1,4-dihydropyridines and 1,1'(1,4-phenylene)bis(1,4-dihydropyridine). Mashaly, M. M.; El-Gogary, S. R.; Kosbar, T. R. Journal of Heterocyclic Chemistry (2014), 51(4), 1078-1085. 
[5] Nonlinear optical material of cyano(acetylamino)cinnamate ester. Hidaka, Takaharu; Nakatani, Hiroyuki; Yamanaka, Kazu. Jpn. Kokai Tokkyo Koho (1991), JP 03206429 A 19910909.

[6] Synthesis, Structure-Activity Relationships and Brain Uptake of a Novel Series of Benzopyran Inhibitors of Insulin-Regulated Aminopeptidase. Mountford, Simon J.; Albiston, Anthony L.; Charman, William N.; Ng, Leelee; Holien, Jessica K.; Parker, Michael W.; Nicolazzo, Joseph A.; Thompson, Philip E.; Chai, Siew Yeen. Journal of Medicinal Chemistry (2014), 57(4), 1368-1377.

[7] AgNO3-Catalysed Intramolecular Cyclization: Access to Functionalized Cyclopentanones and Spiro-Cyclopentanones. Bhoite, Shubhangi P.; Bansode, Ajay H.; Burate, Pralhad A.; Suryavanshi, Gurunath. Asian Journal of Organic Chemistry (2019), 8(10), 1907-1911.

[8] Adenine-Based Coordination Polymers: Synthesis, Structure, and Properties.

Sushrutha, S. R.; Hota, Raghunandan; Natarajan, Srinivasan. European Journal of Inorganic Chemistry (2016), 2016(18), 2962-2974.

[9] Synthesis of 1,7-bis (N-substituted-aminomethyl)-2,8-dihydroxy-Troger's bases and their application in Aldol-Ullmann reaction. Yuan, Rui; Cui, Hao; Chen, Wen; Ren, Xuanxuan; Zhou, Hang; Xu, Hui; Sun, Yawen; Liang, Yanni; Wan, Yu; Liu, Jinjuan; et al. Youji Huaxue (2020), 40(4), 1017-1027.

[10] Synthesis, antitumor activity and molecular docking studies on seven novel thiazacridine derivatives. Almeida, Marcel L.; Viana, Douglas C. F.; Da Costa, Valecia C. M.; Dos Santos, Flaviana A.; Pereira, Michelly C.; Pitta, Maira G. R.; 
Rego, Moacyr J. B. De Melo; Pitta, Ivan R.; Pitta, Marina G. R. Combinatorial Chemistry \& High Throughput Screening (2020), 23(5), 359-368.

[11] Synthesis of 1-[(aryl)(3-amino-5-oxopyrazolidin-4-ylidene) methyl]-2-oxo-1,2dihydroquinoline-3-carboxylic acid derivatives and their breast anticancer activity. Gaber, Ahmed; Alsanie, Walaa F.; Alhomrani, Majid; Alamri, Abdulhakeem S.; ElDeen, Ibrahim M.; Refat, Moamen S. Crystals (2021), 11(5), 571.

[12] Design, synthesis and vasorelaxant evaluation of novel coumarin-pyrimidine hybrids. Amin, Kamilia M.; Awadalla, Fadi M.; Eissa, Amal A. M.; Abou-Seri, Sahar M.; Hassan, Ghaneya S. Bioorganic \& Medicinal Chemistry (2011), 19(20), 6087-6097.

[13] Catalyst study of the Knoevenagel condensation. Prout, Franklin S.; Abdel-Latif, Ata A.; Kamal, Marwan R. Journal of Chemical and Engineering Data (1963), 8(4), 597-9.

[14] Novel copolymers of styrene. 2. Some ring-substituted ethyl 2-cyano-3-phenyl-2propenoates. Kharas, Gregory B.; Molina, Eric S.; Bobot, Bonnie; Bueno, Joanna; Carney, Jason; Chung, J. Y. C.; Krerowicz, Kristen N.; Miller, Tara; Mills, Shana L. I.; Stankovich, Melanie A.; et al. Journal of Macromolecular Science, Part A: Pure and Applied Chemistry (2013), 50(1), 1-5.

[15] SciFinder structure search accessed Dec 16, 2021.

[16] Novel functional copolymers of vinyl acetate: 1. Alkyl ring-substituted ethyl 2cyano-3-phenyl-2-propenoates. Benjamin Y. Killam, Rima T. Barkauskas, Daniel P. Dembiec, Rebecca S. Farrell, Maritza A. Gallego, Kelly A. Kaiser, Michelle L. 
Keeling, Kari Y. Kang, Gretchen R. Verdoorn, and Gregory B. Kharas. ChemRxiv. Cambridge Open Engage Version 1 Sep 06, 2021.

https://doi.org/10.33774/chemrxiv-2021-ksld6

[17] Smith, M. B., March, J. (2001) Addition to Carbon-Hetero Multiple Bonds, In March's Advanced Organic Chemistry; J. Wiley \& Sons: New York, Ch.16, 1225.

[18] Novel Copolymers of Vinyl Acetate with Alkyl and Alkoxy Ring-Disubstituted 2Phenyl-1,1-dicyanoethylenes. G.B. Kharas, S.M. Russell, A. Alshaikh, M.L. Jonathas, A.P. Reddy, A.A. Rusinak, Y. Tsang, R.A.Wilczak, A. Virani, V. Havalad, and E. Hanawa. J. Macromol.Sci., A45 (6) 416-419 (2008).

[19] Characterization of copolymers of vinyl acetate with ethyl $\alpha$-cyanocinnamate. G.B.

Kharas and D.H. Kohn. J. Polym. Sci. Polym. Chem. Ed., 23, 577-582 (1984).

[20] Characterization and physical properties of low molecular weight poly(vinyl acetate) and poly(vinyl alcohol). Toshiaki Sato and Takuji Okaya. Polymer Journal, Vol. 24, No.9, pp 849-856 (1992).

[21] The thermal degradation of poly(vinyl acetate) and poly(ethylene-co-vinyl acetate), Part I: Experimental study of the degradation mechanism. B. Rimez, H. Rahier, G. Van Assche, T. Artoos, M. Biesemans, B. Van Mele. Polymer Degradation and Stability, 93 (2008) 800-810. 